\title{
A Validated Integration Algorithm for Nonlinear ODEs using Taylor Models and Ellipsoidal Calculus
}

Boris Houska
Mario Eduardo Villanueva

\author{
Benoît Chachuat*
}

\begin{abstract}
This paper presents a novel algorithm for bounding the reachable set of parametric nonlinear differential equations. This algorithm is based on a first-discretize-thenbound approach to enclose the reachable set via propagation of a Taylor model with ellipsoidal remainder, and it accounts for truncation errors that are inherent to the discretization. In contrast to existing algorithms that proceed in two phases-an a priori enclosure phase, followed by a tightening phase-the proposed algorithm first predicts a continuous-time enclosure and then seeks a maximal step-size for which validity of the predicted enclosure can be established. It is shown that this reversed approach leads to a natural step-size control mechanism, which no longer relies on the availability of an a priori enclosure. Also described in the paper is an open-source implementation of the algorithm in ACADO Toolkit. A simple numerical case study is presented to illustrate the performance and stability of the algorithm.
\end{abstract}

\section{INTRODUCTION}

The computation of tight enclosures for the solutions of nonlinear ordinary differential equations (ODEs) is used in many rigorous methods in a variety of research fields, including reachability analysis for control systems [3], [20], global optimization of dynamic systems [5], [10], [19], and uncertainty analysis for nonlinear dynamic processes and robust optimal control [9], [11]. Existing methods can be classified into either continuous-time enclosure techniques or time-discretization techniques. In the former class, an auxiliary differential equation is formulated, the solution of which yields an enclosure of the original ODE solutions at any time. Originally proposed to compute interval bounds on the reachable set based on the theory of differential inequalities [26], this approach has been later extended to propagate affine bounds [25], a pair of convex/concave bounds [23], Taylor models [6] as well as ellipsoidal bounds [9].

The focus in this paper is on the second class, referred to as first-discretize-then-bound approach herein. Many validated integration methods for nonlinear ODEs go back to the original work by Moore [16], who presented a simple test for checking the existence and uniqueness of ODE solutions over a finite time step using interval analysis. This test was later used in an algorithm that discretizes the integration horizon into finite steps and proceeds in two phases at each step [17]: (i) determine a step-size and an a priori enclosure of the ODE solutions over this step; then, (ii) propagate a tightened enclosure until the end of the step. In particular,

B. Houska, M.E. Villanueva, and B. Chachuat are with the Centre for Process Systems Engineering, Imperial College London, South Kensington Campus, London SW7 2AZ, UK. b.houska, m.villanueva, b. chachuateic.ac.uk

* Corresponding author. the second phase relies on a high-order Taylor expansion of the ODE solutions in time, which can be evaluated in interval arithmetic or in Taylor model arithmetic [2], [13], [18]. The propagation of convex/concave bounds, using either McCormick relaxations [14] or McCormick-Taylor models [4], has also been proposed [21], [22].

The main contribution of this paper is the development of a first-discretize-then-bound algorithm based on a new bounder for vector-valued functions, namely Taylor models with ellipsoidal remainders. This algorithm features a novel step-size control mechanism that no longer requires the computation of an a priori enclosure as in the classical twophase approach. The paper also comes along with a freely available, open-source implementation of the algorithm in the form of a sub-package in ACADO Toolkit [7].

The remainder of the paper is organized as follows. Sect. II defines the notation and introduces classical Taylor models with interval remainder bounds, before introducing Taylor models with ellipsoidal remainders for vector-valued functions. The ODE bounding problem is then formulated in Sect. III and the new validated integrator is described in Sect. IV. The software implementation is discussed in Sect. V, followed by a numerical case study. Finally, conclusions are drawn in Sect. VI.

\section{BACKGROUND}

This section defines the mathematical notation used throughout the paper and reviews Taylor models with interval remainder bounds. A new bounder type that consists of a Taylor model with ellipsoidal remainder is introduced in Sect. II-A together with a computational approach.

The unit vectors in $\mathbb{R}^{n}$ are denoted by $e_{1}, \ldots, e_{n}$ and we define the vector $e:=(1, \ldots, 1)^{\mathrm{T}}=\sum_{i} e_{i}$. The set of interval vectors in $\mathbb{R}^{n}$ is denoted by

$$
\mathbb{I}^{n}=\left\{[\underline{x}, \bar{x}] \mid \underline{x}, \bar{x} \in \mathbb{R}^{n}, \underline{x} \leq \bar{x}\right\} .
$$

The $k$ th component of an interval vector $X=[\underline{x}, \bar{x}] \in \mathbb{I}^{n}$ is given by $X_{k}:=\left[\underline{x}_{k}, \bar{x}_{k}\right]$, and the norm of $X$ is defined as

$$
\|X\|:=\max _{k} \operatorname{abs}\left(X_{k}\right) \text {, }
$$

with $\operatorname{abs}\left(X_{k}\right):=\max \left\{\left|\underline{x}_{k}\right|,\left|\bar{x}_{k}\right|\right\}$. Moreover, $\operatorname{mid}(X):=\frac{1}{2}(\underline{x}+$ $\bar{x})$ and $\operatorname{rad}(X):=\frac{1}{2}(\bar{x}-\underline{x})$ stand for, respectively, the midpoint and the radius of $X$, evaluated component-wise. Given a continuous function $g: \mathbb{R}^{n} \rightarrow \mathbb{R}^{n}$, the function $g^{\mathbb{I}}: \mathbb{I}^{n} \rightarrow \mathbb{I}^{n}$ is called an $\mathbb{I}$-extension of $g$ if

$$
g^{\mathbb{I}}(X) \supseteq\{g(x) \mid x \in X\}
$$


for all $X \in \mathbb{I}^{n}$. A variety of software implementations are available to compute $\mathbb{I}$-extensions of factorable (a.k.a. treedecomposable) functions.

The set of symmetric and positive semi-definite matrices is denoted by $\mathbb{S}_{+}^{n} \subseteq \mathbb{R}^{n \times n}$. An ellipsoid in $\mathbb{R}^{n}$, centered at the origin, is given by

$$
\mathscr{E}(Q):=\left\{Q^{\frac{1}{2}} v \mid v \in \mathbb{R}^{n}, v^{\mathrm{T}} v \leq 1\right\},
$$

where $Q \in \mathbb{S}_{+}^{n}$ stands for the shape matrix, and we denote the set of ellipsoids in $\mathbb{R}^{n}$ by

$$
\mathbb{E}^{n}:=\left\{\mathscr{E}(Q) \mid Q \in \mathbb{S}_{+}^{n}\right\}
$$

The interval hull of an ellipsoid $\mathscr{E}(Q)$ in $\mathbb{R}^{n}$ is given by

$$
B^{\mathbb{E}}(Q):=[-1,1]\left(\sqrt{Q_{11}} \cdots \sqrt{Q_{n, n}}\right)^{\mathrm{T}} \supseteq \mathscr{E}(Q) .
$$

The set of multivariate polynomials of order $q$ mapping $[-e, e] \subset \mathbb{R}^{m}$ onto $\mathbb{R}^{n}$ is denoted by $\mathbb{P}_{n, m}^{q}$. The image set $\operatorname{Im}(\mathscr{P})$ of a polynomial $\mathscr{P} \in \mathbb{P}_{n, m}^{q}$ is given by

$$
\operatorname{Im}(\mathscr{P}):=\{\mathscr{P}(x) \mid x \in[-e, e]\} .
$$

We assume throughout the paper that a polynomial range bounding function $B^{\mathbb{P}}: \mathbb{P}_{n, m}^{q} \rightarrow \mathbb{I}^{n}$ is available such that

$$
B^{\mathbb{P}}(\mathscr{P}) \supseteq \operatorname{Im}(\mathscr{P}),
$$

for all $\mathscr{P} \in \mathbb{P}_{n, m}^{q}$. In practice, the implementation of such a function can be based, e.g., on LMI methods [12] or other heuristics [2].

The set of Taylor models of order $q$ with interval remainder bounds [4] is denoted by $\mathbb{T} \mathbb{I}_{n, m}^{q}:=\mathbb{P}_{n, m}^{q} \times \mathbb{I}^{n}$. The image of a Taylor model $\mathscr{T}:=[\mathscr{P}, I] \in \mathbb{T} \mathbb{I}_{m, n}^{q}$ is defined as

$$
\operatorname{Im}(\mathscr{T}):=\operatorname{Im}(\mathscr{P}) \oplus I,
$$

where the operator $\oplus$ stands for the Minkowski sum of two sets, and its interval extension is given by

$$
B^{\mathbb{T I}}(\mathscr{T}):=B^{\mathbb{P}}(\mathscr{P}) \oplus I .
$$

Given a $(q+1)$-times continuously differentiable function $g: \mathbb{R}^{n} \rightarrow \mathbb{R}^{n}$, the function $g^{\mathbb{T I}}: \mathbb{T I}_{n, m}^{q} \rightarrow \mathbb{T I}_{n, m}^{q}$ is called a $\mathbb{T I}$-extension of $g$ if

$$
\operatorname{Im}\left(g^{\mathbb{T I}}(\mathscr{T})\right) \supseteq\{g(x) \mid x \in \operatorname{Im}(\mathscr{T})\},
$$

for all $\mathscr{T} \in \mathbb{T I}_{n, m}^{q}$. In practice, a Taylor model of a factorable function can be computed by applying Taylor model arithmetic [4], for which various software implementations have become available in recent years.

\section{A. Taylor Models with Ellipsoidal Remainders}

In analogy to classical Taylor models with interval remainder bounds, we denote by $\mathbb{T E}_{m, n}^{q}:=\mathbb{P}_{n, m}^{q} \times \mathbb{S}_{+}^{n}$ the set of Taylor models of order $q$ with ellipsoidal remainders. The image of such a Taylor model $\mathscr{T}:=[\mathscr{P}, Q] \in \mathbb{T E}_{m, n}^{q}$ is defined as

$$
\operatorname{Im}(\mathscr{T}):=\operatorname{Im}(\mathscr{P}) \oplus \mathscr{E}(Q)
$$

and its interval extension is given by

$$
B^{\mathbb{T E}}(\mathscr{T}):=B^{\mathbb{P}}(\mathscr{P}) \oplus B^{\mathbb{E}}(Q) .
$$

Now, given a $(q+1)$-times continuously differentiable function $g: \mathbb{R}^{n} \rightarrow \mathbb{R}^{n}$, the function $g^{\mathbb{T}}: \mathbb{T}_{n, m}^{q} \rightarrow \mathbb{T I}_{n, m}^{q}$ is called a $\mathbb{T E}$-extension of $g$ if

$$
\operatorname{Im}\left(g^{\mathbb{T E}}(\mathscr{T})\right) \supseteq\{g(x) \mid x \in \operatorname{Im}(\mathscr{T})\},
$$

for all $\mathscr{T} \in \mathbb{T E}_{n, m}^{q}$. As far as the authors are aware, the use of ellipsoidal bounds to enclose the remainder term in the Taylor expansion of a vector-valued function has not been considered in the literature, and no algorithms are available for computing $\mathbb{T} \mathbb{E}$-extension of factorable functions. We propose one such algorithm below.

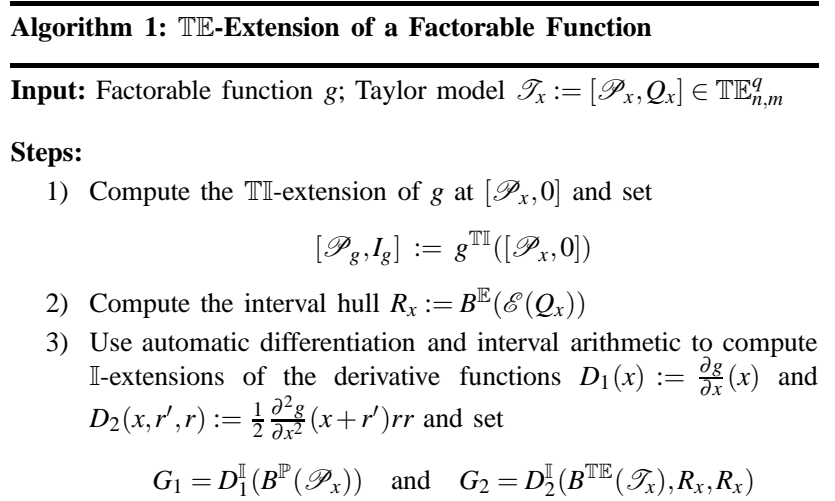

4) Compute $A_{g}:=\operatorname{mid}\left(G_{1}\right)$ and $N_{g}:=I_{g} \oplus\left(G_{1}-A_{g}\right) R_{x} \oplus G_{2}$

5) Find a matrix $Q_{g} \in \mathbb{S}_{+}^{n}$ such that

$$
\mathscr{E}\left(A_{g} Q_{x} A_{g}^{\mathrm{T}}\right) \oplus N_{g} \subseteq \mathscr{E}\left(Q_{g}\right)
$$

Output: $\mathbb{T E}$-extension $g^{\mathbb{T E}}\left(\mathscr{T}_{x}\right):=\left[\mathscr{P}_{g}, Q_{g}\right]$ of $g$ at $\mathscr{T}_{x}$

A number of remarks are in order regarding Algorithm 1.

- The ellipsoidal enclosure of the remainder term of the function $g$ considers a second-order Taylor expansion of the form

$$
g(p+r)=g(p)+\frac{\partial g}{\partial x}(p) r+\frac{1}{2} \frac{\partial^{2} g}{\partial x^{2}}\left(p+r^{\prime}\right) r r,
$$

for $p \in \operatorname{Im}\left(\mathscr{P}_{x}\right)$ and $r^{\prime}, r \in \mathscr{E}\left(Q_{x}\right)$. It follows from basic ellipsoidal calculus [11] that

$$
g(x) \in \operatorname{Im}\left(\mathscr{P}_{g}\right) \oplus \mathscr{E}\left(A_{g} Q_{x} A_{g}^{\mathrm{T}}\right) \oplus N_{g}=\operatorname{Im}\left(\left[\mathscr{P}_{g}, Q_{g}\right]\right),
$$

for any $x \in \operatorname{Im}\left(\mathscr{T}_{x}\right)$. We also note that a higher-order expansion is possible in principle, for instance to further reduce dependencies, yet this comes at the cost of extra computations.

- A way to implement Step 5 is by noting that the inclusion (2) holds upon selecting $Q_{g}$ as

$$
Q_{g}=\frac{1}{\lambda_{0}} A_{g} Q_{x} A_{g}^{\mathrm{T}}+\sum_{i=1}^{n} \frac{1}{\lambda_{i}} \operatorname{rad}\left(N_{g_{i}}\right)^{2} e_{i} e_{i}^{\mathrm{T}}
$$

for any $\lambda_{0}, \lambda_{1}, \ldots, \lambda_{n} \in \mathbb{R}_{+}$with $\sum_{i=0}^{n} \lambda_{i} \leq 1$; see, e.g., [8], [11]. For instance, these scalars can be chosen in 
such a way that the trace of the right-hand side of (3) is minimal, giving

$$
\begin{aligned}
& \lambda_{0}=\frac{\sqrt{\operatorname{Tr}\left(A_{g} Q_{x} A_{g}^{\mathrm{T}}\right)}}{\sqrt{\operatorname{Tr}\left(A_{g} Q_{x} A_{g}^{\mathrm{T}}\right)}+\sum_{k=1}^{n}\left\|\operatorname{rad}\left(N_{g_{k}}\right)\right\|_{2}} \\
& \lambda_{i}=\frac{\left\|\operatorname{rad}\left(N_{g_{i}}\right)\right\|_{2}}{\sqrt{\operatorname{Tr}\left(A_{g} Q_{x} A_{g}^{\mathrm{T}}\right)}+\sum_{k=1}^{n}\left\|\operatorname{rad}\left(N_{g_{k}}\right)\right\|_{2}}, \quad i=1, \ldots, n .
\end{aligned}
$$

\section{Problem Statement}

We consider dynamic systems of the form

$$
\dot{x}\left(t, x_{0}\right)=f\left(t, x\left(t, x_{0}\right)\right), \quad x\left(0, x_{0}\right)=x_{0}
$$

for $t \in[0, T]$, where $x \in \mathbb{R}^{n}$ denotes the state variables; $x_{0} \in$ $X_{0}$ the initial values, with $X_{0} \subseteq \mathbb{R}^{n}$ a compact set; and $f$ : $D \rightarrow \mathbb{R}^{n}$ is a sufficiently often continuously differentiable function on the domain $D:=[0, T] \times \mathbb{R}^{n}$. A solution of (5) corresponding to a given initial value $x_{0} \in X_{0}$ is denoted as $x\left(\cdot, x_{0}\right)$, and the reachable set is defined as $X(t):=\left\{x\left(t, x_{0}\right) \mid\right.$ $\left.x_{0} \in X_{0}\right\}$.

The aim of the following consideration is to devise and implement a numerical algorithm, which constructs a Taylor model $\mathscr{T}_{x}(t) \in \mathbb{T E}_{n, m}^{q}$ such that

$$
\forall t \in[0, T]: \quad X(t) \subseteq \operatorname{Im}\left(\mathscr{T}_{x}(t)\right) .
$$

This construction start at $t=0$ with a Taylor model $T_{x}(0) \in$ $\mathbb{T} \mathbb{E}_{n, m}^{q}$ such that $\operatorname{Im}\left(\mathscr{T}_{x}(0)\right) \supseteq X_{0}$. In particular, the modeling assumption for the set $X_{0}$ is rather general-only compactness is assumed for $X_{0}$, which encompasses the representation of non-convex initial value sets.

It should be noted that this formulation can also deal with parametric ODEs; that is, ODEs whose right-hand side function $f$ depends explicitly on a parameter $p$. In this case, a possible reformulation in the form of (5) involves appending the trivial ODE $\dot{p}(t)=0$ to the dynamic system, thereby regarding $p$ as an extra state variable. While this reformulation is certainly appropriate for our theoretical considerations, we like to mention that it may not be efficient from a computational perspective. In particular, the software implementation described later in Sect. V-A deals explicitly with parametric dependencies as a direct extension of the proposed algorithm.

\section{VAlidated Integrator Propagating TAylor MODELS WITH ELLIPSOIDAL REMAINDERS}

Similar to existing validated integrators for nonlinear ODEs [17], the proposed algorithm considers a Taylor series expansion in time of the ODE solutions. Assuming that $x\left(\cdot, x_{0}\right)$ is the solution of (5) for a given initial value $x_{0}$ until $t$, and provided that the solution $x\left(t+h, x_{0}\right)$ exists for $h \in[0, T-t]$, the application of Taylor theorem for a sthorder expansion gives

$$
x\left(t+h, x_{0}\right)=\sum_{i=0}^{s} h^{i} \phi_{i}\left(t, x\left(t, x_{0}\right)\right)+h^{s+1} \phi_{s+1}\left(\tau, x\left(\tau, x_{0}\right)\right)
$$

for some $\tau \in[t, t+h]$. Here, $\phi_{0}, \phi_{1}, \ldots, \phi_{s+1}: D \rightarrow \mathbb{R}^{n}$ denote the Taylor coefficients of the solution, defined recursively as

$$
\begin{aligned}
\phi_{0}(t, x) & :=x \\
\phi_{i}(t, x) & :=\frac{1}{i}\left[\frac{\partial \phi_{i-1}}{\partial x}(t, x) f(t, x)+\frac{\partial \phi_{i-1}}{\partial t}(t, x)\right],
\end{aligned}
$$

for $i=1, \ldots, s+1$ and for all $(t, x) \in D$.

State-of-the-art validated integrators [17], [18], [22] proceed in two phases, a first phase that determines a step size and an a priori enclosure of the solution trajectory, followed by a tightening of this a priori enclosure in the second phase. In contrast, the algorithm we develop in this paper reverses the order of these two phases, thereby removing the need for an a priori enclosure of the solution and providing a natural mechanism for step-size selection. This procedure is described next for the propagation of Taylor models with ellipsoidal remainders as introduced in Sect. II-A, yet it is readily applied to other types of enclosures such as intervals or standard Taylor models.

- Phase I. Given a Taylor model of the ODE solution at $t$ such that $\operatorname{Im}\left(\mathscr{T}_{x}(t)\right) \supseteq X(t)$, a Taylor model predictor $\mathscr{T}_{x}(t+h)$ of the solution for all $h \in(0, T-t]$ is constructed as

$$
\mathscr{T}_{x}(t+h):=\bigoplus_{i=0}^{s} h^{i} \phi_{i}^{\mathbb{T E}}\left(t, \mathscr{T}_{x}(t)\right) \oplus h \delta[-e, e],
$$

for a pre-specified tolerance $\delta>0$, and where $\phi_{0}^{\mathbb{T E}}, \ldots, \phi_{s}^{\mathbb{T E}}$ are $\mathbb{T E}$-extensions of the Taylor coefficients $\phi_{0}, \ldots, \phi_{s}$.

- Phase II. A step-size $\bar{h}$ is determined, such that the predictor $\mathscr{T}_{x}(t+h)$ is guaranteed to yield a valid enclosure of the reachable set, $\operatorname{Im}\left(\mathscr{T}_{x}(t+h)\right) \supseteq X(t+h)$, for all times $h \in[0, \bar{h}]$. In particular, any feasible point $\bar{h}>0$ of the following optimization problem is suitable:

$$
\begin{aligned}
& \sup _{h>0} h \quad \text { subject to } \\
& \forall \tau \in[t, t+h]:(\tau-t)^{s} \phi_{s+1}^{\mathbb{I}}\left(\tau, B^{\mathbb{T E}}\left(\mathscr{T}_{x}(\tau)\right)\right) \subseteq \delta[-e, e],
\end{aligned}
$$

with $\phi_{s+1}^{\mathbb{I}}$ an $\mathbb{I}$-extension of the Taylor coefficient $\phi_{s+1}$. The validity of the proposed bounding procedure is established in the following theorem.

Theorem 1. Let the function $f$ be $(s+q+1)$-times continuously differentiable and factorable, and let $\mathscr{T}_{x}(t) \in \mathbb{T E}_{n, m}^{q}$ be such that $\operatorname{Im}\left(\mathscr{T}_{x}(t)\right) \supseteq X(t)$. If $\bar{h}$ is a feasible point of the step-size maximization problem (11), then $X(t+h) \subseteq$ $\mathscr{T}_{x}(t+h)$ for all $h \in[0, \bar{h}]$, with $\mathscr{T}_{x}(t+h)$ being given by (10).

Proof. The right-hand side function $f$ being $(s+q+1)$-times continuously differentiable, the Taylor coefficients $\phi_{k}, k=$ $0, \ldots, s$, are at least $(q+1)$-times continuously differentiable. It follows by application of $\mathbb{T E}$-calculus to the Taylor series expansion (7) that $X(t+h) \subseteq \operatorname{Im}\left(\mathscr{T}_{x}(t+h)\right)$ whenever the remainder term $h^{s+1} \phi_{s+1}\left(\tau, x\left(\tau, x_{0}\right)\right)$ is contained in a ball of radius $h \delta$ for all $\tau \in[t, t+h]$. The semi-infinite constraint 
in (11) ensures that the remainder term indeed satisfies this condition on the interval $[0, \bar{h}]$ by construction.

For any practical purposes, it is convenient (and sufficient) to solve the step-size maximization problem (11) approximately. Observe that the semi-infinite constraint in (11) is satisfied when

$$
h^{s} r(h) \subseteq \delta[-e, e],
$$

where the short-hand notation

$$
r(h):=\phi_{s+1}^{\mathbb{I}}\left([t, t+h], B^{\mathbb{T E}}\left(\mathscr{T}_{x}(t+h)\right)\right) \in \mathbb{I}
$$

is used to denote the $\mathbb{I}$-extension of $\phi_{s+1}$. The following simple iterative procedure can be applied to find a feasible step-size.

- Consider the following initial guess $h_{0}$ for the feasible step-size:

$$
h_{0}=\rho\left(\frac{\delta}{\|r(0)\|}\right)^{\frac{1}{s}}
$$

where $0<\rho<1$ is a tuning parameter, e.g., $\rho=\frac{1}{2}$.

- If the inclusion (12) is satisfied with $h_{0}$, select $\bar{h}=h_{0}$.

- Otherwise, iteratively reduce the candidate step-size as $h_{i+1}=\rho h_{i}$, until (12) is satisfied.

Note that this procedure is guaranteed to identify a feasible step-size $\bar{h}>0$ after a finite number of steps, as the left handside expression in (12) shrinks with order $O\left(h^{s}\right)$ whereas the right-hand term is constant and has a non-empty interior.

The $\mathbb{T E}$-based validated integration algorithm is summarized below.

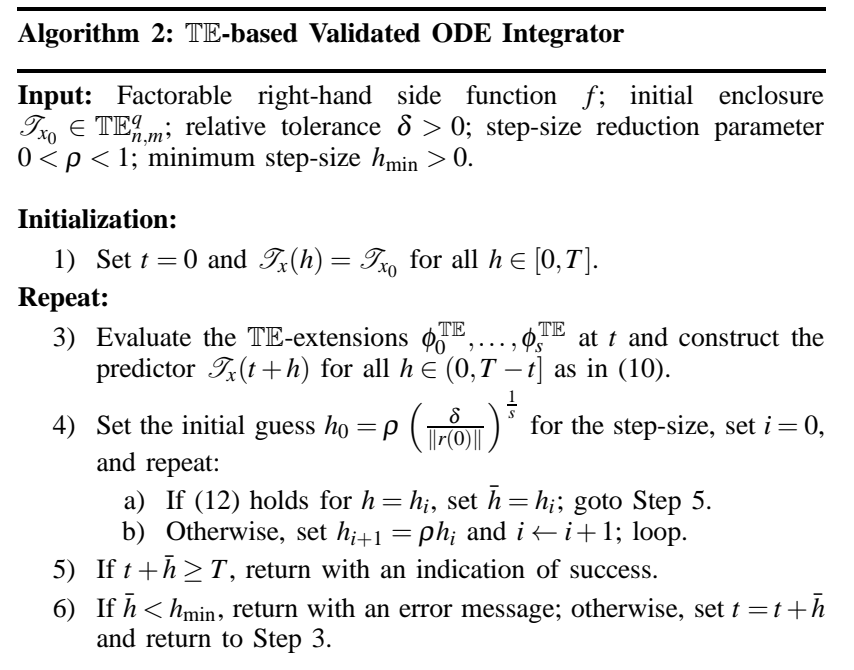

Output: Enclosure function $\mathscr{T}_{x}:[0, T] \rightarrow \mathbb{T E}_{n, m}^{q}$ satisfying (6) until $t$.

Algorithm 2 terminates with an error message if the existence of the reachable set $X(t)$ can no longer be established. Such scenarios cannot be avoided, for instance if a solution trajectory $x\left(t, x_{0}\right)$ fails to exist over the entire horizon $t \in[0, T]$ for a particular initial values $x_{0} \in X_{0}$; this may also occur when the enclosure size blows up due to the wrapping effect. On the other hand, upon successful termination, Algorithm 2 not only yields a valid enclosure of the ODE solution, but also a guarantee that the solution trajectories $x\left(t, x_{0}\right)$ exist for all $x_{0} \in X_{0}=\operatorname{Im}\left(\mathscr{T}_{x_{0}}\right)$.

\section{Implementation and Numerical Case Study}

This section describes the software module that has been developed and illustrates the performance of the proposed algorithm and its implementation.

\section{A. $\mathbb{T E}$-Integrator in ACADO Toolkit}

A C++-based software implementation of Algorithm 2 has been developed, which comes as a sub-package of ACADO Toolkit in a class called Ellipsoidalintegrator. ACADO Toolkit is an open-source collection of numerical algorithms for automatic control and dynamic optimization [7], which is freely available under the LGPL-license and can be downloaded from [1].

Our implementation makes uses of the symbolic userinterface from ACADO Toolkit in order to define the righthand side function. The usual C++ operators '+', '-, '*', '=' etc, as well as a collection of intrinsic functions such as 'pow', 'sqrt', 'exp' etc, are all overloaded in ACADO Toolkit, such that an evaluation tree of the right-side function can be constructed internally-see [7] for more details about the symbolic expressions and tree structured function evaluation in ACADO Toolkit. The internal software design relies heavily on the concept of operator overloading and templates in $\mathrm{C}++$, which allows to keep the code modular and compatible with other software packages. The current default implementation of Taylor model and interval arithmetics reuses classes which have originally been developed as part of the software MC++ [15]. However, the templated class design allows to replace these tools seamlessly, e.g. with a customized Taylor model implementation or an external interval library.

Figure 1 shows an implementation in ACADO Toolkit of the cubic oscillator problem

$$
\begin{aligned}
& \dot{x}_{1}(t)=x_{2}+p_{1}\left(1-x_{1}^{2}-x_{2}^{2}\right) x_{1}, \\
& \dot{x}_{2}(t)=-x_{1}+p_{1}\left(1-x_{1}^{2}-x_{2}^{2}\right) x_{2}-p_{2} x_{2}
\end{aligned}
$$

with fixed parameter values $p_{1}=\frac{1}{10}$ and $p_{2}=\frac{1}{5}$ and initial set

$$
X_{0}=[1.5,2.5] \times[-0.1,0.1] \subseteq \mathbb{R}^{2} .
$$

In this tutorial example and unless otherwise noted, Taylor models of order $q=4$ are used and the Taylor expansion in time of the ODE solution is of order $s=5$-these orders correspond to NQ and NS, respectively, in Figure 1. In executing the algorithm, the initial state interval enclosure $X_{0}$ is first cast as a Taylor model with ellipsoidal remainder:

$$
\mathscr{T}_{x_{0}}=\left[\mathscr{P}_{x_{0}}, 0\right] \in \mathbb{T E}_{2,2}^{4} \quad \text { with } \quad \mathscr{P}_{x_{0}}(y)=\left(\begin{array}{c}
2+0.5 y_{1} \\
0.1 y_{2}
\end{array}\right) \text {. }
$$

The numerical output which is obtained by running the code example in Figure 1 is discussed later in Sect. V-B. 


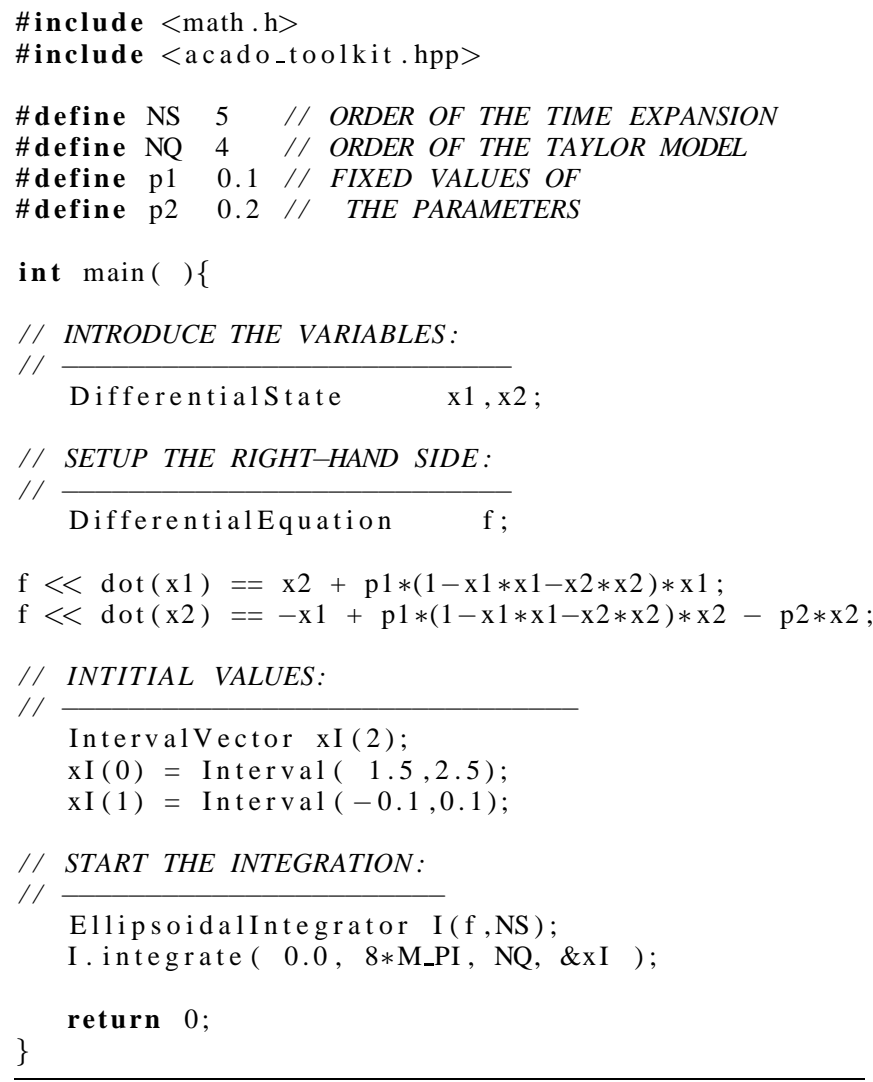

Fig. 1. A tutorial $\mathrm{C}++$ code for using the $A C A D O \mathbb{T E}$-integrator.

The implementation of the $\mathbb{T E}$-integrator in ACADO Toolkit also allows to set several options. For instance, the tolerance $\delta$ can be set to $\delta=10^{-8}$ as follows:

$$
\text { I.set (INTEGRATOR_TOLERANCE, } 1 e-8 \text { ); }
$$

before calling the method integrate $(\ldots)$. More information about settings and options as well as tutorial examples can be obtained from the ACADO Toolkit web-page [1]. A Doxygen-documentation of the class Ellipsoidalintegrator can also be found there.

A current limitation of the implementation in ACADO Toolkit is that numerical round-off errors are not accounted for, as it relies on a non-verified implementation of interval arithmetics. This is usually not critical as the numerical round-off errors are typically in the order of the machine precision, which is hardly critical on standard computers where the machine precision is in the order of $2^{-52}$. However, our current implementation is not yet validated in the strict sense of reliable computing. Nevertheless, since the implementation of the integrator is based on templates, the underlying Taylor model and interval classes can easily be exchanged with validated analogs that would take numerical round-off errors into account.

\section{B. Numerical Case Study}

This section discusses the numerical outer-approximation of the actual reachable set $X(t)$ of the dynamic system (13), for the initial condition set $X_{0}$ in (14). The results obtained by running the $\mathrm{C}++$ code from Figure 1 using $\delta=10^{-8}$, $s=5, q=4$, and $\rho=\frac{1}{2}$ are shown in Figure 2. There, the exact solution set $X(t)$ for the state $x=x_{1}$ and $y=y_{1}$ are represented as a red-shaded area, while the computed bounds are plotted with thin black lines. Clearly, the computed Taylor model $\mathscr{T}_{x}(t)$ encloses the actual reachable set $X(t)$ at all times; that is, $X(t) \subseteq Y(t):=\operatorname{Im}\left(\mathscr{T}_{x}(t)\right)$.

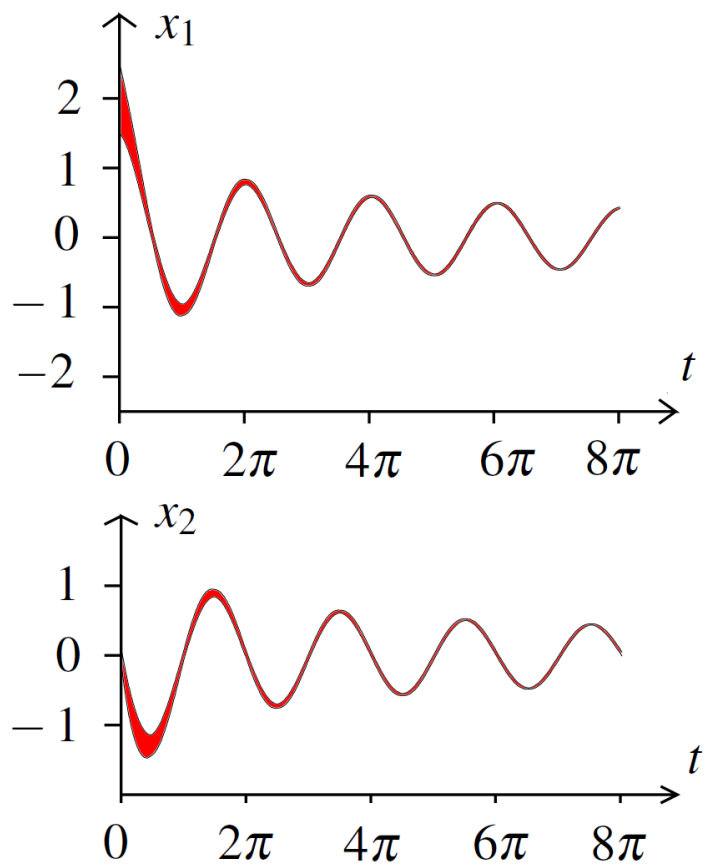

Fig. 2. Projection of the exact reachable set $X(t)$ (red-shaded area) of the first and second component of the state $x(t) \in \mathbb{R}^{2}$. The thin black lines show the upper and lower bounds computed with the $\mathbb{T E}$-integrator over the time horizon $[0,8 \pi]$.

An interesting question at this point relates to how accurately the computed enclosure $Y(t)$ approximates the exact reachable set $X(t)$. This approximation error can be quantified using the Haussdorf distance between the sets $X(t)$ and $Y(t)$ defined as

$$
d_{\mathrm{H}}(X(t), Y(t)):=\inf _{x \in X(t)} \sup _{y \in Y(t)}\|x-y\|_{\infty} .
$$

Figure 3 shows the Haussdorf distance for various Taylor model orders $q \in\{1,2,4,8\}$ along the time horizon. When running the algorithm with Taylor models of order $q=1$, the $\mathbb{T E}$-integrator stops with an error message around $t \approx 34$. This is due to the fact that, in this instance, the inherent stability of the cubic oscillator system (13) is over-powered by the wrapping effect that is inherent to interval and ellipsoidal calculus for the remainder term of the Taylor model. This is also indicated by a dramatic increase in Haussdorf distance between the sets $X(t)$ and $Y(t)$ at $t \approx 34$. Quite remarkably, however, the choice of a Taylor model order of order $q \geq 2$ guarantees that the Haussdorf distance between the sets $X(t)$ and $Y(t)$ remains bounded and stable; that is, the integrator could in principle compute the enclosure set 


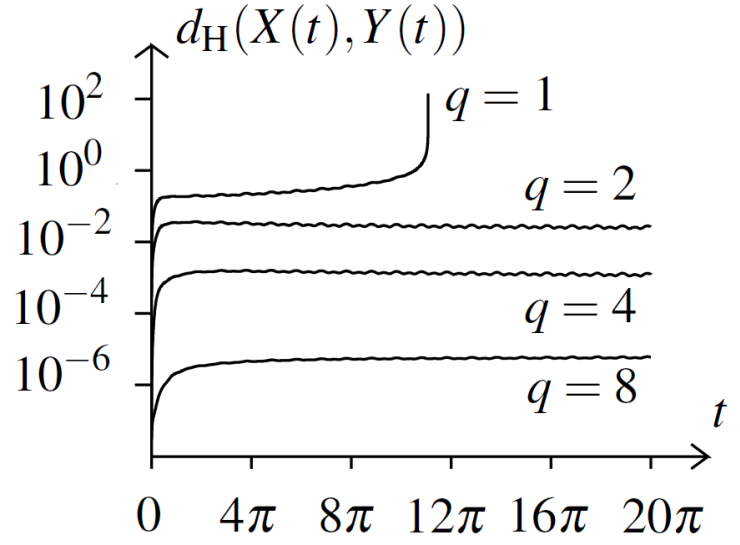

Fig. 3. The Haussdorf distance $d_{\mathrm{H}}(X(t), Y(t))$ between the exact reachable set $X(t)$ and the enclosure $Y(t):=\operatorname{Im}\left(\mathscr{T}_{x}(t)\right)$ computed by the $\mathbb{T} \mathbb{E}$-integrator for different Taylor model orders $q \in\{1,2,4,8\}$.

over an arbitrary long integration horizon. For instance, the Haussdorf distance remains in the order of $10^{-5}$ on the longer horizon $t \in[0,20 \pi]$ when Taylor models of order $q=8$ are used. Nevertheless, a formal proof of the stability of the $\mathbb{T E}$-integrator for sufficiently small initial value sets $X_{0}$ lies beyond the scope of this paper, and will be an interesting topic for future research.

\section{CONCLUSIONS}

This paper has described a novel algorithm for bounding the reachable set of a nonlinear ODE whose right-hand side function is factorable and sufficiently often continuously differentiable. The main idea is summarized in Algorithm 2, which reverses the classical two-phase approach of validated integration in the sense that it first constructs a predictor of the enclosure function and then determines a step-size for which this predictor is valid. This procedure thus leads to a natural step-size control mechanism, whose validity has been established in Theorem 1. Moreover, we have presented a user-friendly $\mathrm{C}++-$ based software implementation of the proposed algorithm in ACADO Toolkit, which is freely available under LGPL license [1]. Preliminary numerical results indicate that the algorithm works well on a simple case study and shows a remarkable stability, as measured by the Haussdorf distance between the exact reachable set and the computed enclosure set. More detailed benchmark tests, including comparisons with other bounding techniques, as well as a formal analysis of the stability properties of the $\mathbb{T E}$-integrator will be investigated as part of future work.

\section{ACKNOWLEDGMENTS}

This paper is based upon work supported by the Engineering and Physical Sciences Research Council (EPSRC) under Grant EP/J006572/1. Financial support from Marie Curie under grant PCIG09-GA-2011-293953 and from the Centre of Process Systems Engineering (CPSE) of Imperial College is gratefully acknowledged. MV thanks CONACYT for doctoral scholarship.

\section{REFERENCES}

[1] ACADO Toolkit Homepage. http://www.acadotoolkit.org, 2009-2013.

[2] M. Berz, and K. Makino. Performance of Taylor model methods for validated integration of ODEs. Lecture Notes in Computer Science, 3732:65-74, 2006.

[3] F. Blanchini and S. Miani. Set-Theoretic Methods in Control. Birkhäuser, 2008.

[4] A. Bompadre, A. Mitsos, and B. Chachuat. Convergence analysis of Taylor and McCormick-Taylor models. Journal of Global Optimization, 57(1):75-114, 2013.

[5] B. Chachuat, A.B. Singer, and P.I. Barton. Global methods for dynamic optimization and mixed-integer dynamic optimization. Industrial \& Engineering Chemistry Research, 45(25):8373-8392, 2006.

[6] B. Chachuat and M.E. Villanueva. Bounding the solutions of parametric ODEs: When Taylor models meet differential inequalities. In I.D.L. Bogle and M. Fairweather, Eds., 22nd European Symposium on Computer Aided Process Engineering, 30:1307-1311, 2012.

[7] B. Houska, H.J. Ferreau, and M. Diehl. ACADO Toolkit - An open source framework for automatic control and dynamic optimization. Optimal Control Applications and Methods, 32(3):298-312, 2011.

[8] B. Houska. Robust Optimization of Dynamic Systems. PhD thesis, KU Leuven, 2011.

[9] B. Houska, F. Logist, J. Van Impe, and M. Diehl. Robust optimization of nonlinear dynamic systems with application to a jacketed tubular reactor. Journal of Process Control, 22(6):1152-1160, 2012.

[10] B. Houska, B. Chachuat. Branch-and-Lift Algorithm for Deterministic Global Optimization in Nonlinear Optimal Control. Journal of Optimization Theory and Applications, in press DOI: 10.1007/s10957013-0426-1.

[11] A.B. Kurzhanski, and P. Valyi. Ellipsoidal Calculus for Estimation and Control. Birkhäuser Boston, 1997.

[12] J.B. Lasserre. Moments, Positive Polynomials and Their Applications. Imperial College Press, 2009.

[13] Y. Lin, and M.A. Stadtherr. Validated solutions of initial value problems for parametric ODEs. Applied Numerical Mathematics, 57:1145-1162, 2007.

[14] G.P. McCormick. Computability of global solutions to factorable nonconvex programs: Part I - Convex underestimating problems. Mathematical Programming, 10:147-175, 1976.

[15] A. Mitsos, B. Chachuat, P.I. Barton. McCormick Based Relaxations of Algorithms. SIAM Journal on Optimization, 20:573-601, 2009.

[16] R.E. Moore, F. Bierbaum. Methods and Applications of Interval Analysis. SIAM, 1979.

[17] N.S. Nedialkov, K.R. Jackson, and G.F. Corliss. Validated solutions of initial value problems for ordinary differential equations. Applied Mathematics \& Computation, 105(1):21-68, 1999.

[18] M. Neher, K.R. Jackson, and N.S. Nedialkov. On Taylor model based integration of ODEs. SIAM Journal on Numerical Analysis, 45:236262, 2007.

[19] I. Papamichail, and C.S. Adjiman. A rigorous global optimization algorithm for problems with ordinary differential equations. Journal of Global Optimization, 24:1-33, 2002.

[20] J.B. Rawlings and D.Q. Mayne. Model Predictive Control: Theory and Design. Nob Hill, 2009.

[21] A.M. Sahlodin, and B. Chachuat. Convex/concave relaxations of parametric ODEs using Taylor models. Computers \& Chemical Engineering, 35:844-857, 2011.

[22] A.M. Sahlodin, and B. Chachuat. Discretize-then-relax approach for convex/concave relaxations of the solutions of parametric ODEs. Applied Numerical Mathematics, 61:803-820, 2011.

[23] J.K. Scott, B. Chachuat, and P.I. Barton. Nonlinear convex and concave relaxations for the solutions of parametric ODEs. Optimal Control Applications \& Methods, 34:145-163, 2013.

[24] J.K. Scott, and P.I. Barton. Improved relaxations for the parametric solutions of ODEs using differential inequalities. Journal of Global Optimization, 57(1):143-176, 2013.

[25] A.B. Singer, and P.I. Barton. Bounding the solutions of parameter dependent nonlinear ordinary differential equations. SIAM Journal on Scientific Computing, 27(6):2167-2182, 2006.

[26] W. Walter. Differential and Integral Inequalities. Springer-Verlag, Berlin, Germany, 1970. 